\title{
IDOSOS RESIDENTES EM MEIO RURAL: SATISFAÇÃO COM O SUPORTE SOCIAL
}

\author{
Catarina Monteiro* \\ Rosa Martins ${ }^{\dagger}$
}

Resumo: Introdução: As múltiplas investigações desenvolvidas sobre o apoio social dos Idosos têm demonstrado, que o apoio percebido e o grau de satisfação com ele, é aquele que tem demonstrado exercer efeitos mais fortes e consistentes na saúde, no bem-estar e na qualidade de vida dos mais velhos.

Objectivo: Avaliar a satisfação dos idosos da amostra com o seu suporte social, e avaliar em que medida as variáveis sóciodemográficas, as variáveis circunstanciais, as variáveis da perceção da saúde e as variáveis sócioculturais influenciam essa satisfação.

Metodologia: Foi realizado um estudo transversal, descritivo, correlacional e não experimental, e de cariz quantitativo. A amostra selecionada é do tipo não probabilística por conveniência, constituída por 130 idosos do concelho de Armamar que recorreram ao Balcão de Proximidade do Projecto 3I's. Os instrumentos de medida utilizados foram um questionário construído para obter informações sobre os dados sociodemográficos, socioculturais, sobre a perceção da saúde e as caraterísticas circunstânciais e a Escala de Satisfação com o Suporte Social.

Resultados: A população estudada é maioritariamente feminina, casada e com uma média de idades de 75,18 anos. Possuem habilitações literárias baixas, (34,6\% analfabetos), auferem de escasso apoio institucional e baixos rendimentos. A grande maioria dos idosos $(96,2 \%)$ reside em casa própria, considera-se autónoma $(85,3 \%)$ e os níveis de satisfação com o suporte social são moderados em todas as dimensões, sendo mais elevados no item satisfação com amizades $(M=16,2)$.

Conclusão: As variáveis com maior influencia na satisfação com o suporte social são: o género, pois os homens possuem níveis de satisfação com o suporte social mais elevados em todas as subescalas

\footnotetext{
* Mestre em Serviço Social, Universidade Católica Portuguesa - Pólo de Viseu

† Professora Coordenadora da Escola Superior de Saúde do Instituto Politécnico de Viseu (rmartins.viseu@gmail.com)
} 
do que as mulheres; menores sentimentos de solidão e a frequência de visitas dos familiares.

Palavras-Chave: Envelhecimento; Meio Rural; Família; Suporte Social.

\begin{abstract}
Introduction: The different studies concerning the elderly people support have shown that the apprehended support and the satisfaction level associated is the one, which has demonstrated to have stronger and consistent impacts in health, wellness and the quality life of the elderly people.

Objective: To evaluate the elderly people sample satisfaction and its social support, as well as to what extent the socio-demographic circumstantial health awareness socio-cultural variables influence this satisfaction.

Methodology: It was carried out a transversal descriptive correlational and none-experimental study, using a quantitative methodology. The used sample is none-probabilistic for convenience, consisting of 130 elderly people living in Armamar County, who resorted to the 3I's Project nearest balcony. The measuring instrument was a questionnaire made for the purpose and the Satisfaction Scale with the Social Support.

Results: The study population was predominantly female and married, with a 75,18 year average age. The education is low $(34.6 \%$ illiterate), receiving poor institutional support and short incomes. The majority (96.2\%) lives in their own home and the most frequent is that the elderly people consider themselves autonomous (85.3\%). The social support satisfaction levels are moderate in all dimensions. However, they are higher in the friendship satisfaction item $(M=$ 16,2).

Conclusion: The variables, which mostly influence satisfaction with the social support are: the gender, because men in all subscales, in comparison with woman have higher satisfaction levels with social support; also less loneliness feelings and the family visit frequency.
\end{abstract}

Keywords: Aging, Rural Area, Family, Social Support.

\title{
1. IDOSOS RESIDENTES EM MEIO RURAL: SATISFAÇÃO COM O SUPORTE SOCIAL
}

\subsection{O Envelhecimento Humano e o Envelhecimento Demográfico}

Os dados demográficos consultados mostram que toda a Europa está a passar por um processo de envelhecimento e de longevidade que acarreta repercussões bastante significativas aos níveis sociofamiliar, socioeconómico e ao nível dos cuidados de saúde (Azeredo, 2011). Na 
verdade, o envelhecimento populacional resulta não só, mas também, do sucesso das políticas de saúde pública e do desenvolvimento socioeconómico.

Este fenómeno contínua a evoluir no século XXI, com o crescimento da proporção de idosos e "grandes idosos", surgindo assim um novo conceito ou seja a $4^{a}$ idade. Perante esta constatação concordamos com a autora em que o envelhecimento demográfico é o fenómeno mais relevante do século XXI nas sociedades desenvolvidas, devido às suas implicações na esfera socioeconómica, para além das modificações que se refletem a nível individual e nos novos estilos de vida (Carrilho, M. et al., 2004).

Para uns o envelhecimento assenta na maior longevidade dos indivíduos, ou seja, no aumento da esperança média de vida. Para outros este conceito está subjacente ao envelhecimento biológico, às alterações a nível psicológico e ainda a determinantes externas. Já o envelhecimento individual é um processo de deterioração endógena e irreversível das capacidades funcionais do organismo. Trata-se de um fenómeno inevitável, inerente à própria vida, é visto como um processo natural, contínuo, diferencial e heterogéneo (Sousa, Figueiredo \& Cerqueira,2004).

Para as mesmas autoras, o envelhecimento foi percebido durante algum tempo como um fenómeno patológico, que era visto como o relacionamento entre o desgaste do organismo e as sequelas das doenças da infância e da idade adulta. Perante isto, afirmam que é um processo multifatorial que leva a uma deterioração fisiológica do organismo do indivíduo. Ainda nos referem que existem duas formas de envelhecimento, o envelhecimento primário (normal) e secundário (ou patológico). Referem-nos assim as diferenças entre eles: o primeiro, envelhecimento normal, aponta para "o processo de diminuição orgânica e funcional, não decorrente de acidente ou doença, mas que acontece, inevitavelmente, com o passar do tempo". O envelhecimento patológico, considera "os fatores que interferem no processo de envelhecimento normal, tais como stress, traumatismo ou doença, suscetíveis de acelerar o processo de envelhecimento primário". As autoras consideram que não é fácil distinguir de uma forma precisa o envelhecimento normal do patológico, sobretudo, "porque determinadas alterações fisiológicas e doenças que acompanham o envelhecimento são, muitas vezes, confundidas com as manifestações do envelhecimento normal" (2004: p.24). 
O Homem é um ser biopsicossocial e cultural em permanente mudança, por isso o envelhecimento tem sido considerado como um fenómeno de cariz individual e multidimensional. Para Azeredo (2011), o processo de envelhecimento possui três componentes: a componente biológica - proveniente da vulnerabilidade crescente e de onde decorre uma probabilidade crescente de morrer, também chamada de senescência; a componente social - relativa aos papéis sociais adequados às expetativas que a sociedade tem para este grupo etário e a componente psicológica - referente à auto-regulação da pessoa no campo de forças, na tomada de decisões e opções, adaptando-se ao processo de senescência e envelhecimento.

De acordo com os dados do INE, o envelhecimento demográfico define-se como o "aumento da proporção das pessoas idosas na população total, em detrimento da população jovem, e/ou da população em idade ativa". Este tem vindo a aumentar consideravelmente em Portugal e dentro dele destacamos o aumento, dos muito velhos "grandes idosos".

Os resultados dos censos 2011 indicam que o índice de envelhecimento do país é de 129, o que significa que Portugal tem hoje mais população idosa do que jovem (INE, 2011).

Em Portugal, a proporção da população com 65 ou mais anos é de $19 \%$ em 2011. Este valor contrasta com os $8 \%$ verificados em 1960, e com os 16\% em 2001. Ou seja, em Portugal existem 2022504 pessoas com 65 ou mais anos, sendo 849188 homens e 1173316 mulheres.

A distribuição da população por sexo é semelhante aos resultados dos censos de 2001. Nos grupos etários com idades mais avançadas predominam as mulheres, relativamente aos homens. No grupo dos 25-64 anos de idade, a percentagem de mulheres é de $28,5 \%$ e a de homens é de $26,6 \%$. Também no grupo etário dos 65 ou mais anos se verifica a preponderância das mulheres, $11 \%$, face aos homens, $8 \%$. Os censos 2011 apuraram que existe o predomínio do número de mulheres face ao de homens. Existe uma maior taxa de mortalidade da população masculina e a menor esperança de vida à nascença dos homens relativamente às mulheres, estes fatores ajudam a explicar estes resultados.

Segundo fonte do INE (2011), a população portuguesa de hoje, é constituída, sobretudo por mulheres (velhice no feminino). 


\subsection{O envelhecimento em meio rural}

Definir "idosos rurais" não é tarefa fácil sendo frequente utilizar-se esta expressão para denominar situações diversas, que vão desde idosos residentes em zonas não urbanas ou em pequenas povoações de menos de 2500 habitantes, a agricultores propriamente ditos vivendo em propriedades mais ou menos isolados (Paúl \& Fonseca, 2005).

Segundo os autores supracitados, muitos são os mitos que rodeiam os idosos habitantes nas aldeias do interior do país: um deles é, que se reformam em pequenas comunidades campestres, solidárias, onde os esperam anos de felicidade e poucas preocupações ou cuidados, pois têm forte apoio de redes familiares, disponíveis e presentes, outro mito é que se pensa que não têm problemas de saúde, nem necessitam de apoios sociais e financeiros, mesmo não sendo ricos, vivem confortavelmente, pois o custo de vida é mais baixo no meio rural. Nem todas as situações relatadas se manifestam ou estão presentes, contudo, no meio rural, o controlo social reside na solidariedade incondicional e na confiança das relações interpessoais, sendo a interdependência entre a pessoa e o meio, em que desempenha um papel, importante na estrutura dos seus valores.

Os idosos rurais vivem longe dos filhos no nosso país, a ideia das famílias rurais alargadas é, nas zonas desertificadas do interior, um verdadeiro mito. Os filhos e netos estão fisicamente ausentes da vida dos idosos, devido à emigração para o estrangeiro ou migração para o litoral/urbano do país. Esta ideia sobre a situação dos idosos rurais também verificada por McLaughlin e Jersen (1998), que refere que a coresidência com filhos adultos é menor nas áreas rurais do que nas urbanas, ainda que possam ter uma rede de suporte maior (citado por Paúl, 2005).

O concelho de Armamar, local da investigação, caracteriza-se por ser um concelho rural, integrando localidades com pouca população, com poucos rendimentos, com recursos que advêm da agricultura e do trabalho à jorna. Centrando-nos no caso específico dos idosos verificamos, que na sua maioria, estão reformados, contudo continuam a trabalhar na agricultura. Constatamos ainda que têm apoio dos familiares mas também dos vizinhos. Apesar de viverem com as necessidades básicas cobertas, existem também algumas exceções que fazem com que os idosos vivam no limiar da pobreza.

De facto como refere Paúl e Fonseca (2005), as aldeias do interior enfrentam grandes desvantagens, pois são na generalidade, envelhecidas e desertificadas. Os mais novos, população ativa, partem para as cidades 
e/ou estrangeiro à procura de uma vida melhor deixando os mais velhos nas suas aldeias por vezes sozinhos.

Neste cenário, os idosos permanecem entregues a si próprios, e/ou apoiados pelo cônjuge/companheiro e caso não existam ficam votados à institucionalização. A prestação de cuidados informais por parte dos descendentes (filhos e até dos netos), ou não tem representação significativa na vida destes idosos, ou se a têm, torna-se por vezes escassa. A nossa experiência do terreno mostra que são poucos os idosos que têm família como apoio, pois o que é realmente representativo nas suas vidas são as trocas de cuidados existentes entre cônjuges. De notar ainda a presença de uma rede de solidariedade e ajuda por parte de amigos e vizinhos, sendo estes na sua maioria pertencentes à mesma faixa etária.

O suporte social em contexto rural tem de facto visibilidade, pois o vizinho é sobretudo o contacto mais permanente de que o idoso dispõe. Porém, a entreajuda altera-se por dificuldades funcionais porque a maioria são idosos e "grandes idosos" que prestam apoio a outros idosos, estando todos em situação semelhante.

\subsection{Definição e Concetualização do Suporte Social}

Após a análise de várias discussões e pontos de vista sobre o envelhecimento, observámos que este surge como um problema social, não sendo meramente do foro individual mas sim um processo complexo e universal que se transforma numa questão social de onde emerge o suporte social.

O suporte social inscreve-se num contexto ecológico, ou seja, é um modo dinâmico e transacional de influência mútua entre o indivíduo e a sua rede de apoio em que são incluídas duas componentes, uma referente ao "apoio", este ligado às atividades dos domínios instrumental e expressivo, e a outra o "social", este que incide, principalmente, no vínculo que a pessoa tem com o meio social que podemos considerar três vertentes: comunitária, de rede social e do relacionamento íntimo (citado por Martins, 2005).

Para que se dê conta da existência de suporte social percebido, devem considerar-se variáveis tais como: grupo de amigos, a frequência de contacto com este grupo e a existência ou não, de amizades mais íntima. O suporte social é importante quer para a saúde quer para a prevenção de doenças, no sentido de promover o bem-estar físico e psicológico (citado por Martins, 2005). 
O conceito de suporte social diz respeito às funções desempenhadas por um grupo (familiares, amigos, vizinhos, entre outros) a favor de um indivíduo, em determinadas situações da sua vida. Desta forma, a análise do apoio social tem em conta a perceção que os indivíduos têm sobre si, que representa a crença generalizada por eles desenvolvida de que são estimados, que os outros se interessam por eles, que estão disponíveis quando precisam e satisfeitos com as relações que têm. O apoio social é um conceito interativo que diz respeito às transações que se estabelecem entre indivíduos (Barrón, 1996).

Podemos então referir que o suporte social é um termo abrangente que diz respeito à quantidade e à coesão das relações sociais que envolvem uma pessoa, à força dos laços estabelecidos, à frequência de contacto e ao modo como é percebido que existe um sistema de apoio. Esse sistema conta com comportamentos que se baseiam em troca de recursos entre pelo menos dois indivíduos: o recetor e o dador. $\mathrm{O}$ dador pode ser um ou mais do que um indivíduo ou ainda uma instituição (Barrón, 1996).

Independentemente do conceito defendido, o suporte social pode ser de dois tipos: formal ou informal. No que diz respeito ao suporte social formal, abrange as organizações sociais formais, como sendo os serviços do Estado, por exemplo programas governamentais, serviços de saúde e os profissionais que trabalham nesses serviços que se organizam de forma a ajudar aos mais necessitados. O suporte social informal está mais direcionado para as relações familiares, de amizade, de vizinhança, entre outras, podendo fornecer ajuda em atividades do dia-a-dia, e para as quais o indivíduo sozinho não conseguiria executar (Martins, 2005).

Nesta lógica de entendimento e para que sejam resolvidas necessidades básicas de sobrevivência, é fulcral a existência de um apoio instrumental, que se baseia quer em termos materiais/bens e/ou serviços. Não menos importante é o apoio de convívio social uma vez que leva uma pessoa a ter, junto de outras, atividades de lazer ou culturais, que não só a distraiam como a podem aliviar das tensões acumuladas no quotidiano e ao mesmo tempo estar envolvida na sociedade, de forma adquirir ou reafirmar a sua identidade social e evitar o isolamento (Martins, 2005).

Ao contrário do que é esperado, as redes de suporte social, podem significar perigo, mesmo que as intenções do dador sejam de beneficiar o recetor, ou seja, o suporte social pode trazer efeitos negativos. É o caso do apoio dado permanentemente, de forma unilateral (Ramos, 2002).

Como evidencia Barrón (1996), em algumas situações, os efeitos negativos do suporte social oferecido não são úteis nem adequados ao 
receptor, por diversas razões, nomeadamente: efeitos negativos produzidos pela tarefa do cuidador. Um efeito negativo que é de salientar é a falta de auto-estima por parte das pessoas idosas com dependência, que lhes vai causar transtorno saber que não pode retribuir o apoio recebido, o que pode levar à insatisfação da pessoa idosa. Os participantes da rede social ,por vezes, não têm em conta as vontades do idoso e, é possível perceber muitas vezes uma perspectiva que parece considerar a pessoa idosa como alguém sem consciência. Isso causa uma falta de controlo do idoso.

Em geral, estes efeitos negativos das relações sociais contribuem para exacerbar problemas de saúde entre os idosos.

O que é importante não é a quantidade de relações sociais, mas sim, a qualidade dessas relações. Isto pode ser explicado pelo caráter voluntário das relações com pessoas que não são parentes e com os efeitos positivos que este carácter pode causar. O idoso, à medida que vai avançando na idade vai sofrendo perdas na sua vida de relação, quer por morte (ou afastamento) de alguns membros que constituem a sua rede social, quer porque começa a ter limitações funcionais que o impedem de manter assiduamente estas relações. O seu mundo de relação vai-se, assim, tornando mais pequeno dado que enquanto mais novo este era percecionado como grande, contudo a pessoa é dotada de grande plasticidade, conseguindo ter capacidade para compensar estas perdas.

Assim, é importante que um indivíduo crie uma rede social, alargada e diversificada que o mantenha socialmente ativo em idade avançada. Esta rede deve envolver outros elementos, para além das amizades ligadas à sua ocupação, pois quando se reforma, estas podem diminuir drasticamente.

\section{INVESTIGAÇÃO EMPÍRICA}

A componente metodológica é um aspecto essencial em qualquer processo de investigação, uma vez que serve de "guia" durante o desenvolvimento da pesquisa. Todos estes procedimentos metodológicos e suas tomadas de decisão são fundamentais para garantir a fiabilidade e qualidade dos resultados obtidos e consequente resposta à problemática em estudo (Fortin, 1999).

Neste contexto a questão de partida da investigação foi definida da seguinte forma: Será que os idosos do Concelho de Armamar, a viver em meio rural, estão satisfeitos com o seu suporte social? 
Para a sua consecução optámos por uma metodologia que se enquadra no paradigma dos estudos quantitativos, do tipo não experimental, transversal, cuja população-alvo inclui os idosos que residem no concelho de Armamar. Perante a impossibilidade de aceder ao grupo completo, participaram os idosos que recorreram aos serviços do "Balcão de Proximidadet" do concelho de Armamar. Optamos por um tipo de amostragem não probabilística por conveniência que é constituída por 130 Idosos, sendo que 85 pertencem ao sexo feminino e 45 ao sexo masculino.

Assim sendo, o objetivo geral desta investigação consiste em: avaliar a satisfação dos idosos da amostra, com o seu suporte social, e em que medida as variáveis sociodemográficas, circunstanciais, da perceção da saúde e as socioculturais influenciam essa satisfação.

Como objetivos específicos enumeramos os seguintes:

- Identificar as características sociodemográficas dos idosos;

- Conhecer as variáveis circunstanciais dos idosos Inquiridos;

- Conhecer a perceção que estes idosos têm sobre o seu estado de saúde;

- Descrever as atividades socioculturais praticadas pelos idosos e suas condicionantes;

- Identificar a satisfação dos idosos, com o seu suporte social;

- Analisar correlações existentes entre variáveis sociodemográficas, circunstanciais, perceção da saúde e socioculturais e a satisfação com o suporte social.

Relativamente ao tipo de estudo em questão, podemos dizer que se trata de um estudo descritivo, visto que pretendemos descrever características de uma determinada população (idosa) e/ou fenómeno (satisfação com o suporte social), e estabelecer relação entre as variáveis, optámos assim por uma investigação descritiva correlacional (Fortin, 2009).

Optámos por um questionário por nós produzido, constituído por partes em que tentamos averiguar as variáveis sociodemográficas, circunstanciais, perceção da saúde e socioculturais e a satisfação com o suporte social; e também pela aplicação de uma escala, a Escala de Satisfação com o Suporte Social ESSS- Scale for Social Support (Wethingson e Kessler, 1986; Versão portuguesa de Ribeiro, 1999). Esta escala é composta por 15 itens, que se dividem em quatro subescalas:

* Serviço semelhante a balcão móvel de saúde, onde os idosos e famílias podiam recurrer a serviços básicos de saúde e de ação social. 
Satisfação com a amizade (SA) que contém 5 itens, Intimidade (IN) com 4 itens, Satisfação com a família (SF), de 3 itens e Atividades Sociais (AS) com 3 itens. Apresenta como formato de resposta uma escala de 5 pontos que varia entre 1 (Concordo totalmente) a 5 (Discordo totalmente). O resultado é obtido através do somatório de todos os itens, variando os resultados entre 15 a 75 , sendo que os resultados mais elevados significam maior suporte social.

Uma hipótese é um enunciado formal das relações previstas entre duas ou mais variáveis, é uma previsão de resposta para o problema da investigação, que pode ser passível de ser declarada verdadeira ou falsa (Fortin, 2000).

As hipóteses que formulamos para a nossa pesquisa são de dois tipos: complexas e simples.

Hipóteses complexas:

H1- - Existe associação entre as variáveis sociodemográficas e a satisfação dos Idosos com o suporte social.

H2- - Existe associação entre as variáveis circunstanciais e a satisfação dos Idosos com o suporte social.

H3- - Existe associação entre a perceção da saúde e a satisfação dos Idosos com o suporte social.

H4- - Existe associação entre as variáveis socioculturais e a satisfação dos Idosos com o suporte social.

\section{Hipóteses Simples:}

H1a - Existe associação entre o género e a satisfação dos Idosos com o suporte social.

H1b - Existe associação entre a idade e a satisfação dos Idosos com o suporte social.

H1c - Existe associação entre o estado civil e a satisfação dos Idosos com o suporte social.

H1d - Existe associação entre habilitações literárias a satisfação dos Idosos com o suporte social.

H1e - Existe associação entre o rendimento mensal e a satisfação dos Idosos com o suporte social.

H1f - Existe associação entre a satisfação com o rendimento mensal e a satisfação dos Idosos com o suporte social.

H2a - Existe associação entre a variável "com quem vive" e a satisfação dos Idosos com o suporte social.

H2b - Existe associação entre o sentir-se sozinho no seu dia-a-dia e a satisfação dos Idosos com o suporte social. 
H2c - Existe associação entre o número de horas que passa sozinho e a satisfação dos Idosos com o suporte social.

H2d - Existe associação entre a frequência das visitas e a satisfação dos Idosos com o suporte social.

H3a - Existe associação entre a percepção da saúde (autonomia) e a satisfação dos Idosos com o suporte social.

$\mathrm{H} 3 \mathrm{~b}$ - Existe associação entre a percepção da saúde (prática de exercício físico) e a satisfação dos Idosos com o suporte social.

\section{DISCUSSÃO DOS RESULTADOS}

Recordamos que este estudo foi levado a cabo com o propósito de se conhecer a perceção que os idosos têm sobre o suporte social que auferem. As hipóteses colocadas foram no sentido de tentar responder, em parte, a esta questão. Mais concretamente no sentido de perceber se, o facto, dos idosos viverem em meio rural influencia ou não a perceção do suporte social.

Verificamos assim que os idosos vivem maioritariamente, com o cônjuge e/ou sozinhos sendo, poucos os que residem com os filhos. De notar que são as mulheres as que vivem mais sozinhas e contrariamente os homens vivem mais frequentemente com o cônjuge. Estes resultados derivam, possivelmente, do facto de as mulheres ficarem viúvas mais cedo e os homens contraírem novos casamentos.

Pudemos constatar que no concelho de Armamar os idosos ainda são visitados, na sua maioria pelos familiares, sobretudo nas épocas festivas, contudo, ficou igualmente claro que as visitas dos vizinhos/amigos são peças fundamentais que compõem o seu quotidiano. Contrariamente à maioria, ainda encontramos quem responda frontalmente não ser visitado por ninguém, dado que engrossa as fileiras dos que defendem que a solidão é um sentimento muito presente nos idosos.

As respostas dadas sobre os apoios recebidos demonstram que, derivam essencialmente dos familiares, seguindo-se aqueles cuja perceção é a que não recebem nenhum apoio e só depois aparecem os vizinhos/amigos por quem se consideram apoiados. De facto as famílias continuam a constituir os principais apoios dos idosos embora os vizinhos em muitas circunstâncias sejam também peças fundamentais, uma vez que, em especial nos meios rurais, sejam considerados parte integrante da família. 
Os idosos da nossa amostra avaliaram o seu suporte social como Satisfatório. A comparação por géneros mostra uma avaliação mais positiva por parte dos indivíduos do sexo masculino.

Ao questionarmos os participantes sobre "se se considera feliz" verificamos que a maioria dos idosos se considera Medianamente Feliz.

No que diz respeito aos dados sobre os níveis de satisfação dos idosos com o suporte social avaliados pelas dimensões da escala centrando-nos nos resultados da amostra total, verificamos que os níveis de satisfação são moderados em todas as dimensões, contudo são mais elevadas no item satisfação com amizades $(\mathrm{M}=16,2)$ seguindo-se intimidades $(\mathrm{M}=$ $11,4)$ e a satisfação com a família $(\mathrm{M}=9,6)$. $\mathrm{O}$ valor mais baixo encontra-se na satisfação com as atividades sociais $(\mathrm{M}=5,8)$.

Os participantes que sabem ler e escrever possuem níveis mais elevados de suporte social da subescala Satisfação com amizades; Intimidades e Satisfação com família. No entanto, os participantes que não sabem ler e escrever têm níveis mais elevados de satisfação na subescala atividades sociais, ou seja, os idosos analfabetos da nossa amostra, envolvem-se nas atividades da comunidade onde existe a promoção de socialização.

Segundo Azeredo (2011:93) "nas famílias unigeracionais o cuidador principal é, geralmente o cônjuge, funcionando os descendentes como cuidadores secundários, sendo para aqueles um suporte afetivo e instrumental importante". Esta autora ainda salienta que, tal acontece devido aos anos de convivência e ao mútuo conhecimento entre o casal, o cônjuge é a pessoa que está mais apto para o papel de cuidador.

Refira-se que os participantes em que a frequência de visitas dos familiares é inferior ou igual a uma semana, possuem médias mais elevadas na satisfação com as amizades, com a família, nas intimidades e nas atividades sociais. Portanto, a frequência das visitas dos familiares aos idosos são preditivos na determinação da satisfação com o suporte social.

De acordo com Trivalle (2002) não são só as qualidades do suporte social recebidas que estão relacionadas com o bem-estar, uma vez que o suporte social dado aos outros também aumenta a saúde mental. O nível de reciprocidade entre o suporte recebido e o suporte dado revela um nível satisfatório de autonomia, servindo esta capacidade como um moderador do impacto da estratégia direta e do apoio dado sobre o bemestar dos idosos. Os idosos que enfrentam de forma direta os seus problemas, recebem e dão apoio são os que assumem maior bem-estar. Tal situação contribuiu para a mudança na conceção dos idosos como 
sendo um recetor passivo de ajuda, levando a que seja visto também como sujeito ativo na promoção do seu bem-estar.

\section{CONCLUSÃO}

A investigação que nos propusemos desenvolver permitiu-nos comprovar o fato de o envelhecimento ser na verdade um processo complexo e que muitos idosos não estão ainda despertos para as mudanças que este comporta.

Tal como foi referido inicialmente, a população residente em Portugal sofreu um aumento no número de idosos, sendo este evidente na região Norte do país, região esta, que inclui o concelho de Armamar, onde residem os nossos inquiridos. O índice de envelhecimento do país é de 129 , o que significa que tem hoje mais população idosa do que jovem.

A literatura tem apontado para o aumento do envelhecimento populacional, a implicação deste na aquisição de doenças mentais e do crescente isolamento, solidão, abandono dos mais velhos e falta de apoios sociais. Desta forma, tornou-se premente o estudo da perceção do suporte social dos idosos, deste concelho, a fim de o conhecermos para posteriormente podermos promover atividades e, mudanças de comportamentos, que envolvam estes indivíduos, famílias e comunidade em geral, de forma a diminuir os efeitos negativos do envelhecimento.

Hoje em dia, não é suficiente pensar na terceira idade apenas com o objetivo de conseguir retardar o envelhecimento e aumentar o tempo de vida, mas sim, prolongar a duração da vida com qualidade e bem-estar.

Um dos mitos que rodeiam os idosos habitantes nas aldeias do interior do país em que um deles é, que se reformam em pequenas comunidades campestres, solidárias, onde os esperam anos de felicidade e poucas preocupações ou cuidados, pois têm forte apoio de redes familiares, disponíveis e presentes. Outro centra-se na ideia de que não têm problemas de saúde, nem necessitam de apoios sociais e financeiros, mesmo não sendo ricos, vivem confortavelmente, pois o custo de vida é mais baixo no meio rural (Azeredo, 2011). Após a nossa investigação podemos concordar com a autora em algumas considerações, não são mitos, o facto de os idosos rurais terem um apoio familiar mais próximo e que realmente não necessitam de tantos recursos financeiros como podem precisar os idosos a viver nas cidades. Mas isto acontece porque os idosos, mais especificamente, no concelho de Armamar, ainda cultivam os campos para seu sustento e geograficamente os filhos que vivem no concelho ainda terem o cuidado de visitar os pais. Existindo ainda no 


\section{Catarina Monteiro e Rosa Martins}

concelho um vasto número de emigrantes que deixam os seus idosos sozinhos, visitando-os só uma vez no ano. Contudo, necessitam de apoios sociais e de saúde tanto ou mais como os que vivem nas cidades.

Os idosos rurais também necessitam de um suporte social coeso e definido. Quando referenciamos suporte social este é, muitas vezes associado a um tipo de ajuda, formal ou informal, a algo que suporta uma pessoa que está em carência, ou a uma fonte de recursos a que essa pessoa recorre para diminuir as suas necessidades e dificuldades. O suporte social é considerado como uma variável importante, quer para a saúde como para a prevenção da doença.

O suporte social em contexto rural tem de facto visibilidade, pois o vizinho é sobretudo o contacto mais permanente de que o idoso dispõe. Porém a entreajuda altera-se por dificuldades funcionais porque a maioria são idosos e "grandes idosos" que prestam apoio a outros idosos, estando todos em situação semelhante, existindo assim uma troca de vivências, por vezes conjuntas de solidariedade entre os seus habitantes.

O estudo mostrou também que a família tem um papel preponderante na satisfação dos idosos com o seu suporte social, pois o apoio familiar está intrinsecamente ligado aos seus efeitos positivos. Quando os idosos têm perceção e satisfação com o suporte social que recebem, adquirem um sentimento de que são amados, sentindo-se seguros para lidar com os problemas do dia-a-dia e com uma auto-estima mais elevada.

Pretendeu-se que esta investigação venha a contribuir para a compreensão de alguns fatores psicossociais, pertinentes para a caracterização e compreensão desta faixa etária, no interior da região Norte do País, mais propriamente no concelho de Armamar.

No que respeita a existência de associação entre as variáveis sociodemográficas e a satisfação com o suporte social, os dados obtidos apontam para a não existência de relação na totalidade das dimensões. Contudo, o género tem influência na satisfação com as amizades, pois constatamos que os homens possuem níveis de satisfação com o suporte social mais elevado do que as mulheres. O estado civil também tem alguma influência pois os indivíduos que vivem acompanhados possuem níveis de satisfação com o suporte social mais elevados do que os que vivem sozinhos.

Como forma de intervenção podemos apontar a promoção de estilos de vida saudável, através da criação de interesses e atividades de estímulo à saúde física e mental, bem como à ocupação de tempos livres. Esta intervenção pode ser conseguida através da criação de espaços de debate e de lazer nos espaços rurais, dirigidos essencialmente a esta faixa etária. 
Pensamos ser também urgente proceder à alteração das políticas sociais permitindo, o acesso a todos os idosos aos serviços de apoio social, sobretudo ao apoio emocional ou psicológico, apoio na manutenção da saúde e de integração social.

Com base no estudo feito e nos resultados apresentados, estamos convictos que os objetivos inicialmente propostos foram conseguidos, no entanto temos consciência que se trata de uma investigação com algumas limitações regularmente inerentes aos estudos, porém destacamos as limitações temporais.

Esperamos e acreditamos que o estudo aqui apresentado, e as reflexões efectuadas sobre os resultados obtidos, possam constituir um ponto de referência para a compreensão de múltiplos factores no processo de envelhecimento, bem como da influência dos factores psicossociais e da importância do suporte social na fase da velhice. Desta forma, esperamos contribuir para que o estudo do envelhecimento se torne mais conhecido para ser melhor vivido.

Propomos ainda a criação de uma equipa técnica multidisciplinar, que intervenha diretamente na vida quotidiana destes idosos sobretudo porque falamos de idosos a residir em aldeias perdidas no interior Norte de Portugal, onde os recursos são bastante escassos e muitas vezes agravados pelas dificuldades de comunicação. Queremos destacar o bom exemplo e trabalho da equipa técnica do Projecto 3I's, Projecto de Desenvolvimento Social de Armamar, que apesar do excelente trabalho, que levou a cabo neste contexto geográfico e social, teve um tempo curto de vigência.

Com a finalização da presente investigação esperamos estimular a consciência para a realidade vivida e percebida pelos idosos, pois só assim será possível promover o tão desejado e proclamado Envelhecimento ativo e da solidariedade entre Gerações que o ano de 2012 promoveu.

\section{BIBLIOGRAFIA}

Azeredo, Z. (2011). O idoso como um todo... Viseu: Psicosoma.

Barrón, A. (1996). Apoyo Social: Aspectos teóricos y aplicaciones. Madrid: SigloVeintiuno España Editores.

Carrilho, M., et al. (2004). A situação demográfica recente em Portugal. Revista de Estudos Demográficos, 36, 127-152. Lisboa: INE.

Fortin, M. (1999). O Processo de investigação: da concepção à realização. Loures: Lusociência. 
Fortin, M. (2000). O processo de investigação: da concepção à realização. $2^{\mathrm{a}}$ Edição. Loures: Lusociência.

Fortin, M. (2009). Fundamentos e etapas no processo de investigação. Loures: Lusodidacta.

INE. (2011). Censos 2011 - Resultados Provisórios. Lisboa: Instituto Nacional de Estatística, I.P. Consultado em 03 de Janeiro de 2012. Disponível em http://censos.ine.pt/xportal/xmain? $x$ pid=CENSOS \&xpgid=censos 20 11_apresentacao

Martins. R. (2005). A relevância do apoio social na velhice. Millenium: Revista do Instituto Superior Politécnico de Viseu, 31, 128-134. Consultado em 04 de Dezembro de 2010. Disponível em http://www.ipv.pt/millenium/millenium31/9.pdf

Paúl, M. (2005). Envelhecimento activo e redes de suporte social. Sociologia. Pp: 275-287.

Paúl, M., \& Fonseca, A. (2005). Envelhecer em Portugal. Lisboa: Climepsi Editores.

Ramos, M. (2002). Apoio social e saúde entre idosos. Sociologias, 7 (4), 156-175.

Sousa, L., \& Cerqueira, M. (2004). Envelhecer em familiar: Os cuidadores familiares na velhice. Porto: Ambar.

Trivalle, C. (2002). Gerontologie preventive: elements de prevention du vieillissement patholigique. Paris: Masson. 